\title{
The Human Epidermal Growth Factor Receptor 2 Screening Tests for Breast Cancer Suggested by the New Updated Recommendation of the American Society of Clinical Oncology/College of American Pathologists Will Involve a Rise of the In-Situ Hybridization Tests for the European Laboratories of Pathology
}

\author{
Christian Garbar, Aude-Marie Savoye, Corinne Mascaux, Eva Brabencova, and Hervé Curé \\ Biopathology and Oncology Departments, Institut Jean Godinot, Unicancer, 1 rue du Général Koenig CS80014, \\ 51726 Reims Cedex, France \\ Correspondence should be addressed to Christian Garbar; christian.garbar@reims.unicancer.fr
}

Received 13 February 2014; Accepted 20 March 2014; Published 22 April 2014

Academic Editors: G. Gatti, G. E. Kim, and T. Yokoe

Copyright (C) 2014 Christian Garbar et al. This is an open access article distributed under the Creative Commons Attribution License, which permits unrestricted use, distribution, and reproduction in any medium, provided the original work is properly cited.

\begin{abstract}
Aims. The differences between the 2007 and the 2013 ASCO/CAP HER2 guidelines have been compared. We also discussed the potential consequences in our pathological practice. Material and Methodology. 189 HER2 fluorescence in situ hybridisation (FISH) tests were performed from 1016 preliminary HER2 immunohistochemical tests (IHC). All cases were reviewed and reclassed following the 2007 and 2013 ASCO/CAP recommendations. Results. The 2013 version decreased false-negative IHC (3/118 versus $1 / 54, P=$ ns) and created more $2+$ IHC $(40 / 186$ versus $89 / 186, P=0.001)$ or more $3+$ IHC $(9 / 186$ versus $39 / 186, P=0.001)$. One false-positive IHC was described for the 2013 version $(0 / 9$ versus $1 / 39, P=n s)$. Equivocal FISH was reduced $(8 / 186$ versus $2 / 186$, $P=\mathrm{ns}$ ). An estimation based on our data for 1000 patients showed a rise of our FISH tests for the control of $2+$ IHC (180 tests for the 2007 version versus 274 tests for the 2013 version or FISH work overflow is $+52 \%$ ) and for the control of $2+/ 3+$ IHC ( 300 for the 2007 version versus 475 for the 2013 version or FISH work overflow is $+58 \%$ ). Conclusions. The new 2013 ASCO/CAP guidelines have detected more HER2 positive cases but have increased the number of FISH tests.
\end{abstract}

\section{Introduction}

In Europe, the breast cancer incidence is 464.000 cases representing the most common cause in women [1]. Most of them are treated following the oestrogens receptors (ER), progesterone receptors (PR), and epidermal growth factor receptor type 2 (HER2) status [2].

The human epidermal growth factor receptor 2 (HER2) was first discovered in 1984. The HER2 gene encodes a transmembrane tyrosine kinase receptor protein involved in proliferation, tumoral invasion, angiogenesis, apoptosis, and metastasis. For equivalent clinical and pathological stage, patients benefiting of anti-HER2 therapy showed a better prognosis than HER2-negative patients [3].
In the literature, the HER2 gene is classically amplified in $9 \%$ to $74 \%$ of human breast cancers (mean $22 \%$ ) [4]. In 1998, the trastuzumab treatment of positive HER2 invasive breast cancer associated with its companion immunohistochemistry test (IHC) was approved by the Food and Drug Administration.

Consequently, the HER2 status is actually based on IHC triage tests and chromogenic or fluorescence in situ hybridization (FISH) controlling the equivocal IHC results. The choice of IHC is based on its low cost, easy preservation of staining slides, and use of a familiar routine histology. Its disadvantages are the sensible preanalytic issues such as duration and type of fixation, intensity of antigen retrieval, type of antibody, lack of positive internal control signal, 
and difficulties of reproducible scoring. The FISH advantages are the presence of internal control and the more objective scoring system. The disadvantages are the higher cost and the time-consuming to perform the technique. The potential cause of false-positive FISH is to confuse in situ carcinoma area with invasive component. False-negative FISH results are rare and are often the consequence of the tumoral heterogeneity [4].

To improve the efficiency of the HER2 screening, the American Society of Clinical Oncology (ASCO) and the College of American Pathologists (CAP) edited, in 2007, recommendations to the HER2 pathological diagnosis, according to an equilibrated economic/benefits strategy of the anti-HER2 treatment [5].

One of disadvantages of this 2007 version was the true possibility to make false-negative IHC tests and nonnegligible number of equivocal FISH.

To answer to this reality, an important update of the ASCO/CAP HER2 recommendations has been recently published to improve the accuracy of the HER2 test [6].

The aim of this retrospective work is to compare the main differences between the 2007 and the 2013 ASCO/CAP HER2 guidelines and the potential consequences in our routine practice.

\section{Materials and Methodology}

2.1. Patient Population. Between January 2012 and June 2013, 217 HER2 fluorescence in situ hybridisation (FISH) tests were performed in our institution from 217 women (age: $62.5 \pm 13.9$ years) presenting with breast cancer. All cases were selected by preliminary HER 2 immunohistochemical tests presenting from 1016 cases. FISH was indicated for equivocal results (2+: $175 / 217(80.6 \%)$ ) or for quality control (0 or 1+: 10/217 (4.6\%) and $3+: 32 / 217(14.7 \%))$. For this study and according to the literature recommendations, 28 FISH tests were excluded because the fixation was nonoptimal $(n=14)$ and crush artefacts $(n=6)$, the tumoral tissue was too small to be correctly interpreted $(n=4)$, and the diagnosis was not appropriated such as in situ carcinoma with microinvasion $(n=4)[5,6]$. Finally, 189 cases were available for this study: 104 surgical samples and 85 core needle aspiration biopsies (CNB). The main characteristic of patients was the following: pT1b: 10/80 (12.5\%), pT1c: 35/80 (43.7\%), pT2-3: 35/80 (43.7\%), pN0: 36/72 (50.0\%), pN1-2: 36/72 (50.0\%), SBR1: 17/171 (9.9\%), SBR2: 87/171 (50.8\%), and SBR3: 67/171 (39.1\%). The prevalence of positive HER2 in our population of 1016 women calculated with the 2007 recommendations was of $15.1 \%$.

2.2. Histological Procedures. All surgical specimens were initially fixed in $4 \%$ buffered formaldehyde solution between 6 and 48 hours and then imbedded in paraffin and cut to $4 \mu \mathrm{m}$. The slides were stained with a classical haematoxylineosin stain to perform the initial diagnosis.

Immunohistological staining was performed with the Dako Autostainer Link 48 immunostaining system (Dako Glostrub, Denmark) using HER2 primary antibodies (clone

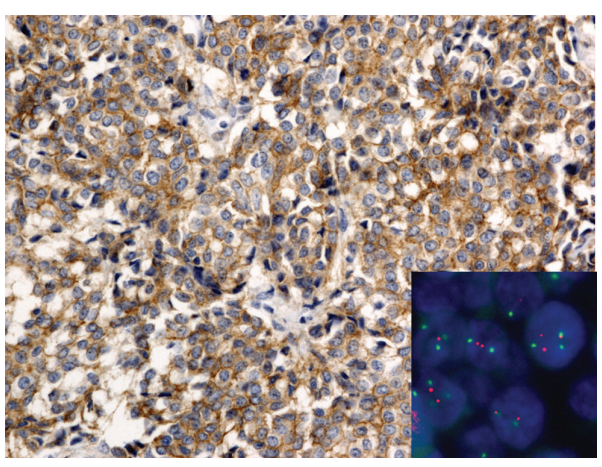

FIGURE 1: An 89-year-old woman with ductal breast carcinoma. IHC classified 1+ with the 2007 ASCO/CAP recommendation and 2+ with the 2013 ASCO/CAP version. Ratio between HER2 (red) and CEN-17 (green) is 2. FISH is equivocal for the 2007 system and negative for the 2013 because the mean of HER2 copy is inferior to 4. HER2 IHC (Clone A0485, Dako, 200x magnification) and HER2 FISH (HER2 IQFISH pharm DX, Dako, 1000x magnification).

A0485, Dako) and according to the manufacturer's instructions $[7,8]$.

HER2 IHC of all cases was confirmed by a rapid FISH technique using HER2/C17 probes (HER2 IQFISH pharm DX, Dako), according to the manufacturer's instructions. Briefly, specimen was denaturised at $66^{\circ} \mathrm{C}$ for 10 minutes. The hybridisation was performed for 90 minutes at $45^{\circ} \mathrm{C}$ simultaneously for HER2/Texas Red labelled DNA probe and CEN-17/FITC labelled DNA probe using a hybridizer device (Dako). We used a fluorescence microscope with appropriate filters (NIKON, Japan).

2.3. Quantification. All HER2 IHC were blindly and independently reviewed by CG and CM according to both the 2007 and the 2013 guidelines of the College of American Pathologists [5, 6]. Discordant results were secondarily discussed and the consensual diagnosis has been retained for the final result.

Briefly, for 2007 version, a positive HER2 test was defined as follows (Figures 1, 2, 3, and 4).

(1) Positive IHC (3+) stains or more than $30 \%$ of tumor cells present intense and uniform circumferential membrane staining with a homogeneous and continuous cell positivity giving a "chicken wire pattern." Incomplete or pale membrane staining is ignored.

(2) Or a positive FISH presents a ratio between HER2 gene and chromosome 17 signals superior to 2.2.

Equivocal test is characterized as

(1) IHC presenting a weak to moderate complete membrane in superior to $10 \%$ tumor cells $(2+)$;

(2) FISH ratio between 1.8 and 2.2.

A negative result is an IHC staining of $0 / 1+$ and a FISH ratio inferior to 1.8 .

For 2013 version, a positive HER2 test was defined as follows (Figures 1, 2, 3, and 4). 


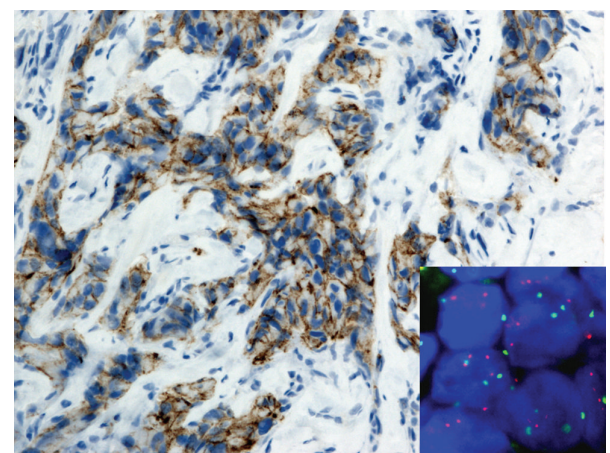

FIGURE 2: A 68-year-old woman with ductal breast carcinoma. IHC classified 1+ with the 2007 ASCO/CAP recommendation and 2+ with the 2013 ASCO/CAP version. Ratio between HER2 (red) and CEN-17 (green) is 1.9. FISH is equivocal for the 2007 system and equivocal for the 2013 because the mean of HER2 copy is 4.5. HER2 IHC (Clone A0485, Dako, 200x magnification) and HER2 FISH (HER2 IQFISH pharm DX, Dako, 1000x magnification).

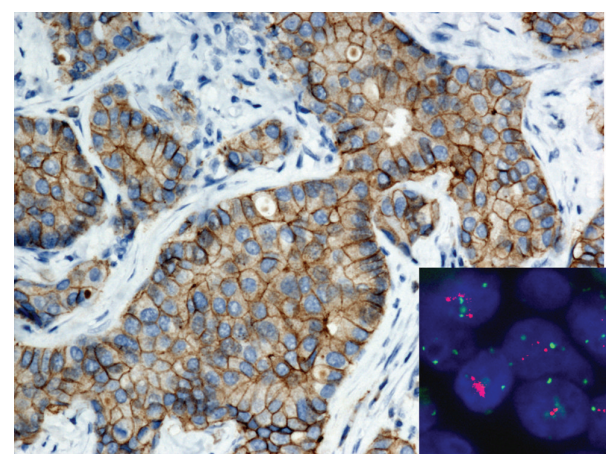

FIGURE 3: A 66-year-old woman with ductal breast carcinoma. IHC classified 2+ with the 2007 ASCO/CAP recommendation and 3+ with the 2013 ASCO/CAP version. Ratio between HER2 (red) and CEN-17 (green) is 1.9. FISH is equivocal for the 2007 system and positive for the 2013 because the mean of HER2 copy is 6.2. HER2 IHC (Clone A0485, Dako, 200x magnification) and HER2 FISH (HER2 IQFISH pharm DX, Dako, 1000x magnification).

(1) Positive IHC within more than $10 \%$ of tumor cells presents intense and uniform complete circumferential membrane staining with a homogeneous and continuous cell positivity giving a "chicken wire pattern" (3+). Incomplete or pale membrane staining is ignored.

(2) Or a positive FISH presents a ratio between HER2 gene and chromosome 17 signals superior or equal to 2.0

(3) Or a positive FISH presents an average of HER2 copy superior or equal to 6.0 .

Equivocal test is characterized as

(1) positive IHC within less than or equal to $10 \%$ of tumor cells presenting intense circumferential membrane staining $(2+)$;

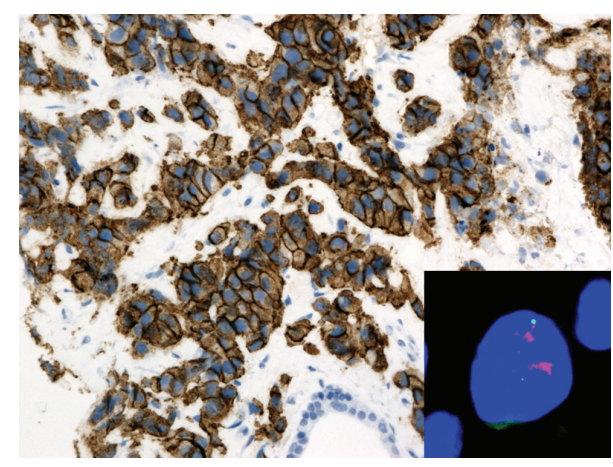

Figure 4: A 91-year-old woman with ductal breast carcinoma. IHC classified 2+ with the 2007 ASCO/CAP recommendation and $3+$ with the 2013 ASCO/CAP version. Ratio between HER2 (red) and CEN-17 (green) is 5.3. FISH is positive for the 2007 system and positive for the 2013. HER2 IHC (Clone A0485, Dako, 200x magnification) and HER2 FISH (HER2 IQFISH pharm DX, Dako, 1000x magnification).

(2) positive IHC within more than $10 \%$ of tumor cells presenting weak/moderate incomplete membrane staining (2+);

(3) FISH ratio inferior to 2.0 with an average of HER2 copy superior or equal to 4.0 and inferior to 6.0 signals.

A negative result is an IHC staining of $0 / 1+$ and a FISH ratio $<2.0$ and an average HER2 copy inferior to 4.0.

2.4. Statistics. ANOVAs tests were performed for parametric results and Fisher's exact test for nonparametric data. Kappa sensitivity and specificity statistical tests were evaluated by the Analyse-it 2.30 (Leeds, UK) and Excel 2003 (Microsoft Corp., Redmond, Washington, USA) programs. A $P$ value $<0.05$ was considered significant.

\section{Results}

In the 2007 guidelines (Table 1), 137 cases were negative in which there were 3 false-negative IHC. Equivocal IHC (2+) was HER2 gene amplified on FISH in 52.5\% (21/40). No false-positive IHC has been observed for $3+$. These results illustrated the weakness of the 2007 guidelines creating some false-negative results that have been discussed in the 2013 version [6]. 8 FISH tests were considered as equivocal, mainly for $1+$ and $2+$ but not for $3+$.

The 2013 version (Table 1) created more positive results for the HER2 IHC+FISH test (33/186 versus 39/186, $P=$ ns). There is a decrease of false-negative IHC (3/186 versus $1 / 186, P=$ ns). This recent 2013 version showed more $2+$ IHC (40/186 versus $89 / 186, P=0.001)$. For these $2+$ IHC, amplification of FISH was only of $14 / 89$ or $15.7 \%$. We have also observed more $3+$ IHC (9/186 versus $39 / 186, P=0.001)$. One false-positive IHC was described for the 2013 version (0/9 versus $1 / 26, P=\mathrm{ns})$. Equivocal FISH was also reduced (8/186 versus $2 / 186, P=$ ns). 
TABLE 1: Comparison between the 2007 recommendations system and the 2013 updated recommendations.

\begin{tabular}{ccccccccc}
\hline & \multicolumn{9}{c}{ FISH } \\
& $\mathrm{E}$ & $\mathrm{N}$ & $\mathrm{P}$ & Total & $\mathrm{E}$ & $\mathrm{N}$ & $\mathrm{P}$ & Total \\
\hline IHC & & & & & & & & \\
$0+$ & 0 & 19 & 0 & $\mathbf{1 9}$ & 0 & 17 & 0 & $\mathbf{1 7}$ \\
$1+$ & 7 & 108 & 3 & $\mathbf{1 1 8}$ & 0 & 53 & 1 & $\mathbf{5 4}$ \\
$2+$ & 1 & 18 & 21 & $\mathbf{4 0}$ & 1 & 74 & 14 & $\mathbf{8 9}$ \\
$3+$ & 0 & 0 & 9 & $\mathbf{9}$ & 1 & 1 & 24 & $\mathbf{2 6}$ \\
Total & $\mathbf{8}$ & $\mathbf{1 4 5}$ & $\mathbf{3 3}$ & $\mathbf{1 8 6}$ & $\mathbf{2}$ & $\mathbf{1 4 5}$ & $\mathbf{3 9}$ & $\mathbf{1 8 6}$ \\
\hline
\end{tabular}

The data illustrates the increasing of 2+ IHC (resp., for 2007 and 2013 guidelines of 40 versus 89 ), $3+$ IHC (9 versus 26 ), and positive HER2 FISH ( 33 versus 39 ). There are less equivocal FISH results (8 versus 2 ) and more false-positive $3+$ IHC (0 versus 1$)$.

FISH: fluorescence in situ hybridization, IHC: immunohistochemistry, E: equivocal, $\mathrm{N}$ : negative, and $\mathrm{P}$ : positive main changes being the increasing of $2+$ and 3+ IHC or amplified HER2 FISH.

TABLE 2: Comparison and correspondence between 2007 and 2013 recommendations systems for immunohistochemistry.

\begin{tabular}{ccccccc}
\hline & & \multicolumn{5}{c}{2013 guidelines } \\
& & $0+$ & $1+$ & $2+$ & $3+$ & Total \\
\hline \multirow{4}{*}{2007 guidelines } & $0+$ & 17 & 2 & 0 & 0 & $\mathbf{1 9}$ \\
& $1+$ & 0 & 52 & 65 & 1 & $\mathbf{1 1 8}$ \\
& $2+$ & 0 & 0 & 24 & 16 & $\mathbf{4 0}$ \\
& $3+$ & 0 & 0 & 0 & 9 & $\mathbf{9}$ \\
& Total & $\mathbf{1 7}$ & $\mathbf{5 4}$ & $\mathbf{8 9}$ & $\mathbf{2 6}$ & $\mathbf{1 8 6}$ \\
\hline
\end{tabular}

The data shows the changes of $1+$ IHC to $2+$ IHC $(n=65)$ and the $2+$ IHC to $3+$ IHC $(n=16)$, respectively, between the 2007 and the 2013 recommendations.

TABLE 3: Comparison and correspondence between 2007 and 2013 recommendations systems for fluorescence in situ hybridization.

\begin{tabular}{cccccc}
\hline & & \multicolumn{4}{c}{2013 guidelines } \\
& & $\mathrm{E}$ & $\mathrm{N}$ & $\mathrm{P}$ & Total \\
\hline \multirow{3}{*}{2007 guidelines } & $\mathrm{E}$ & 1 & 3 & 4 & $\mathbf{8}$ \\
& $\mathrm{N}$ & 1 & 145 & 2 & $\mathbf{1 4 8}$ \\
& $\mathrm{P}$ & 0 & 0 & 33 & $\mathbf{3 3}$ \\
& Total & $\mathbf{2}$ & $\mathbf{1 4 8}$ & $\mathbf{3 9}$ & $\mathbf{1 8 6}$ \\
\hline
\end{tabular}

Kappa test between the 2 ASCO/CAP versions is excellent: 0.96 (95\% CI: 0.91-1.0). There are less equivocal results with the 2013 recommendations. E: equivocal, $\mathrm{N}$ : negative, and P: positive.

Note: the decrease of equivocal FISH of the 2013. Two negative cases became positive because the means of HER2 gene were more than 4 copies.

Table 2 illustrated that an important part of $1+$ IHC of 2007 version became $2+$ IHC in the 2013 version $(65 / 118$, $55 \%)$. Similarly, some $2+$ of the 2007 version were reclassified in $3+$ IHC in the 2013 version (16/40, 40\%).

Table 3 demonstrated the decrease of equivocal FISH and the rise of positive results when we used the 2013 version. When equivocal amplification results were discarded, the Kappa test between the 2 versions was excellent and of 0.96 (95\% CI: 0.91-1.0).
The global Kappa test between IHC and FISH was also good for 2007 version of 0.85 (95\% CI of $0.68-1.0)$ and excellent for the 2013 version of 0.96 (95\% CI 0.91-1.0).

Table 4 calculated an estimation of the 2 guidelines for 1000 women: for the 2007 version, 180 FISH tests were performed to control $2+$ IHC or 300 when the $2+$ and $3+$ IHC were controlled. In comparison with the 2013 version, 274 FISH tests were performed to control 2+ IHC and 475 when $2+$ and $3+$ were controlled. The FISH work overflow for the 2013 version was, respectively, $+52 \%$ for the controls of $2+$ IHC and $+58 \%$ for the controls of $2+$ and $3+$ IHC.

\section{Discussion}

This retrospective study, based on selected revised IHC and FISH cases, has demonstrated that the 2013 ASCO/CAP guidelines of HER2 evaluation are better than the $2007 \mathrm{rec}-$ ommendations. Indeed, the Kappa agreement test between IHC and FISH was higher for the 2013 version (0.96) than for the 2007 (0.85). No false-positive result was observed with the 2007 system. On the contrary, with the 2013 guidelines, we created some false-positive results. To reduce this rate of falsepositive result, some European countries such as Belgium, Germany, or Sweden perform an automatic FISH on all 2+ and $3+$ IHC results [9]. The aim of this health politics is based on the fact that false-positive HER2 can lead to treating patients by a potentially toxic and ineffective anti-HER2 therapy. Nevertheless, other authors such as Ross argued that the impact of false-positive HER2 is less important on patients outcome than false-negative results [10]. Also, Rydn et al. calculated that $14.3 \%$ of invasive breast cancers were HER2 positive. FISH analysis of $2+$ confirmed $12 \%$ of amplification and $90 \%$ for 3+ [9]. In other words, they found $10 \%$ of false-positive HER2 IHC that can be partially explained by the HER2 tumor heterogeneity [11].

Dendukuri et al. discuss the cost effectiveness to test all HER2 2+ and 3+ IHC confirmed by FISH. They found a rate of $282 \mathrm{FISH}$ tests on 1000 women (95\% CI: 142-444) [12]. Here, we calculated, for 1000 women, a rate of $300 \mathrm{FISH}$ tests (180 HER 2+ IHC and 120 HER2 3+ IHC) for the 2007 guidelines and 475 FISH tests for the 2013 system that would be retested by FISH (Table 4).

Although our regional HER2 prevalence of 15.1 seems relatively low, recent German multicentric data from 18.081 women has calculated the average HER2-positivity rate of $16.7 \pm 3.2 \%$ [13]. Also in Sweden the prevalence was $14.3 \%$ [9]. These positive HER2 rates were similar to our results.

We also demonstrated that the number of $2+$ IHC of the 2013 guidelines was increased twice than that of the 2007, and consequently FISH tests increased similarly (resp., 89/186 for the 2013 version versus $40 / 186$ for the 2007 version). In our experience, low HER2 prevalence involves a tendency of pathologists to reclassify HER2/IHC $1+$ to $2+$ in order to reduce the number of potential false-negative IHC tests. In other words, the present 2013 recommendation is reassuring for pathologists. Another advantage of the 2013 guidelines is to decrease the number of equivocal FISH amplifications. This problem was recently debated by the French Association of Pathologists that has proposed a HER2 evaluation system, 
TABLE 4: Example of FISH work overflow calculated for 1000 women.

\begin{tabular}{|c|c|c|c|c|c|c|}
\hline Indications of the FISH test & $\begin{array}{l}2007 \\
\text { guidelines } \\
2+\mathrm{IHC} \\
\end{array}$ & $\begin{array}{c}2007 \\
\text { guidelines } \\
2+\text { and } 3+\end{array}$ & $\begin{array}{c}2013 \\
\text { guidelines } \\
2+ \\
\end{array}$ & $\begin{array}{c}2013 \\
\text { guidelines } \\
2+\text { and } 3+\end{array}$ & $\begin{array}{c}2013 \\
\text { guidelines } \\
\text { All cases } \\
\end{array}$ & \\
\hline $\mathrm{IHC} 0+/ 1+$ & 700 & 700 & 525 & 525 & - & (1) \\
\hline IHC $2+$ & 180 & 180 & 274 & 274 & - & (2) \\
\hline IHC $3+$ & 120 & 120 & 201 & 201 & - & (3) \\
\hline $\mathrm{FISH}+$ & 36 & 36 & 43 & 236 & 236 & (4) \\
\hline $\begin{array}{l}\text { Number of false-positive IHC } 3+/ 1000 \\
\text { cases }\end{array}$ & 0 & 0 & 8 & 0 & - & (5) \\
\hline Prevalence of HER2 & $15.6 \%$ & $15.6 \%$ & $24.4 \%$ & $23.6 \%$ & $23.6 \%$ & \\
\hline Number of IHC performed & 1000 & 1000 & 1000 & 1000 & 0 & \\
\hline Number of FISH tests performed & 180 & 300 & 274 & 475 & 1000 & \\
\hline $\begin{array}{l}\text { Net FISH overwork with } 2007 \text { as } \\
\text { reference }(\%) \text { for FISH controlling } 2+ \\
\text { IHC }\end{array}$ & $0 \%$ & $+66 \%$ & $+52 \%$ & $+163 \%$ & $+455 \%$ & \\
\hline $\begin{array}{l}\text { Net FISH overwork with } 2007 \text { as } \\
\text { reference (\%) for FISH controlling 2+ and } \\
3+\text { IHC }\end{array}$ & $-40 \%$ & $0 \%$ & $-8 \%$ & $+58 \%$ & $+233 \%$ & \\
\hline
\end{tabular}

FISH work overflow calculations for 1000 women when we use the 2007 or the 2013 ASCO/CAP recommendations with HER2 FISH as control of 2+ IHC or $2+/ 3+$ IHC (system used in some European countries). The last column illustrated a system of HER2 screening only based on HER2 FISH as used in the USA. (1) For the 2007 system, 350 cases of $0+$ IHC and 350 cases of $1+$ IHC are based on the $69.8 \%$ of our $0 / 1+$ IHC of our clinical data. Table 1 showed that 54/118 cases (about $50 \%$ ) of $1+$ became $2+$ with the 2013 system, about $350 * 0.5=175$ cases.

(2) Table 1 showed that $16 / 40(40 \%)$ of the $2+$ became $3+$ for the 2013 system. For 1000 cases, $180 \times 0.4=81$ cases. For 2013 system, $180-81+175=274$.

(3) For the 2013 system, 3+ are the sum of 120 cases of $3+$ IHC of the 2007 system and 81 cases of the $2+$ IHC of the 2007 system that changes in $3+$ IHC in the 2013 system. The total of $3+$ IHC for the 2013 system is 201 cases.

(4) For the 2007 system, $20 \%$ of our $2+$ are amplified (20/40) and, for 2013 system, $15.7 \%$ of $2+$ are amplified (14/89).

In the column of 2013 guidelines of FISH for $2+$ and $3+, 240$ is calculated for 201 IHC $3+-8$ false-positive cases (see 5) +43 cases of $2+$ IHC positive FISH.

(5) Table 1: $1 / 25$ or $4 \%$ is the rate of false-positive rate for $3+$ IHC of the 2013 system. For 1000 cases, there are $2013+$ IHC or 8 false-positive cases $(201 \times 0.4)$.

quite similar to the 2013 CAP system for FISH [14]. This GEFPICS's approach is interesting and shows a good correlation with 2013 ASCO/CAP guidelines (excellent Kappa of 0.98, data not shown).

The problem of the HER2/IHC variability is well known. Indeed, HER 2 protein is affected by preanalytic steps of the histological procedure such as the tumor cold ischemia, the duration of tissue formaldehyde fixation, the tissueprocessing technique, and the paraffin embedding temperature $[4,15]$. In a series of 421 cases comparing IHC and FISH with the 2007 guidelines, Vergara-Lluri et al. found a Kappa coefficient of 0.89 , similar to our observations. Interestingly, most of their false-negative tests were due to underestimating the IHC 1+, supporting the 2013 guidelines [16].

About FISH, HER2 DNA is more resistant than HER2 protein to tissue alterations caused by preanalytical processes: false-negative results of FISH are rare and false-positive results are associated with the confusing of in situ component in place of the invasive carcinoma. Consequently, Ross et al. proposed FISH method as the primary HER2 screening. This strategy could be justified by an increasing of the accuracy and a more precise use of targeted therapy [17]. Identically, Sauter et al. pointed out that the standardization of IHC in paraffin-embedded tissue is problematic leading to $2 \%$ to $8 \%$ of false-negative HER2 in IHC 0/1 [18]. These authors argue that primary FISH testing is more cost effective than the evaluation of all IHC-positive patients associated with
FISH. The robustness of HER2 FISH was also illustrated by Grimm et al. [19] that described $4 \%$ of discordant tests presenting a positive IHC and a negative FISH and explained this discordance more by interpretative errors than by a technical error. The USA pathological practice currently uses FISH as the primary HER2 screening test and IHC as the control test. This methodology does not correspond to the European reality where IHC is the primary HER2 screening test.

The main critic of our study is the selection of patients: 28 cases were discarded, particularly because a technical defect potentially causes false HER2 results, well documented in the literature such as crush artefacts or retraction and small size of tumoral sample [20]. Nevertheless, we did not have the pretention to give epidemiological data and the aim of this study was only to evaluate the impact of the new 2013 recommendation in our European practice. It seems clear that the main change will be an increasing of twice FISH. To reduce this technician time-consuming caused by the FISH work overflow, we used the new rapid FISH technology developed by Dako. This technique was recently evaluated on TMA and approved as similar as the classical FISH method [21]. We point out that, to the best of our knowledge, this study is the first using this new rapid IQFISH Dako system on routine material.

In conclusion, targeted therapy for breast cancer is the new challenge for a personalized medicine and the HER2 
FISH or IHC test is the best companion test of reference [22]. Standardization is necessary to treat optimally all candidate patients for a targeted therapy. The new 2013 ASCO/CAP guidelines give us an efficient and robust methodology to realize this aim. Nevertheless, these recommendations could create an increasing of the global cost of the HER2 screening for the laboratories of pathology, mainly caused by the increase of FISH tests, more expensive than IHC and more time-consuming. Finally, further large study could surely need to estimate the true cost effective/benefit ratio of the new ASCO/CAP HER2 guidelines on our European practice.

\section{Conflict of Interests}

The authors declare that there is no conflict of interests regarding the publication of this paper.

\section{References}

[1] J. Ferlay, E. Steliarova-Foucher, J. Lortet-Tieulent et al., "Cancer incidence and mortality patterns in Europe: estimates for 40 countries in 2012," European Journal of Cancer, vol. 49, no. 6, pp. 1374-1403, 2013.

[2] A. Goldhirsch, E. Winer, A. Coates et al., "Personalizing the treatment of women with early breast cancer: highlights of the St Gallen Internalional Expert Consensus on the primary therapy of early breast cancer," Annals of Oncology, vol. 24, no. 9, pp. 2206-2223, 2013.

[3] D. J. Slamon, B. Leyland-Jones, S. Shak et al., "Use of chemotherapy plus a monoclonal antibody against her2 for metastatic breast cancer that overexpresses HER2," The New England Journal of Medicine, vol. 344, no. 11, pp. 783-792, 2001.

[4] J. S. Ross, E. A. Slodkowska, W. F. Symmans, L. Pusztai, P. M. Ravdin, and G. N. Hortobagyi, "The HER-2 receptor and breast cancer: ten years of targeted anti-HER-2 therapy and personalized medicine," The Oncologist, vol. 14, no. 4, pp. 320368, 2009.

[5] A. C. Wolff, M. E. H. Hammond, J. N. Schwartz et al., "American Society of Clinical Oncology/College of American Pathologists guideline recommendations for human epidermal growth factor receptor 2 testing in breast cancer," Journal of Clinical Oncology, vol. 25, no. 1, pp. 118-145, 2007.

[6] A. Wolff, E. Hammond, D. Hicks et al., "Recommendations for human epidermal growth factor receptor 2 testing in breast cancer: American society of clinical oncology/college of American pathologists clinical practice guideline update," Journal of Clinical Oncology, vol. 31, pp. 3997-4013, 2013.

[7] A. M. Gown, L. C. Goldstein, T. S. Barry et al., "High concordance between immunohistochemistry and fluorescence In situ hybridization testing for HER2 status in breast cancer requires a normalized IHC scoring system," Modern Pathology, vol. 21, no. 10, pp. 1271-1277, 2008.

[8] T. A. Thomson, M. M. Hayes, J. J. Spinelli et al., "HER-2/neu in breast cancer: interobserver variability and performance of immunohistochemistry with 4 antibodies compared with fluorescent In situ hybridization," Modern Pathology, vol. 14, no. 11, pp. 1079-1089, 2001.

[9] L. Rydn, M. Haglund, P.-O. Bendahl et al., "Reproducibility of human epidermal growth factor receptor 2 analysis in primary breast cancer A national survey performed at pathology departments in Sweden," Acta Oncologica, vol. 48, no. 6, pp. 860-866, 2009.

[10] J. Ross, "Saving lives with accurate HER2 testing," American Journal of Clinical Pathology, vol. 134, pp. 183-184, 2010.

[11] E. Perez, M. Press, A. Dueck et al., "Immunohistochemistry and fluorescence In situ hybridization assessment of HER2 in clinical trials of adjuvant therapy for breast cancer (NCCTG N9831, BCIRG 006, BCIRG 005)," Breast Cancer Research and Treatment, vol. 138, pp. 99-108, 2013.

[12] N. Dendukuri, K. Khetani, M. McIsaac, and J. Brophy, “Testing for HER2-positive breast cancer: a systematic review and costeffectiveness analysis," Canadian Medical Association Journal, vol. 176, no. 10, pp. 1429-1434, 2007.

[13] H. Choritz, G. Büsche, and H. Kreipe, "Quality assessment of HER2 testing by monitoring of positivity rates," Virchows Archiv, vol. 459, no. 3, pp. 283-289, 2011.

[14] F. Penault-Llorca, A. Vincent-Salomon, J.-P. Bellocq et al., “Update of the GEFPICS' recommendations for HER2 status determination in breast cancers in France," Annales de Pathologie, vol. 30, no. 5, pp. 357-373, 2010.

[15] A. C. Wolff, M. E. H. Hammond, J. N. Schwartz et al., "American Society of Clinical Oncology/College of American Pathologists guideline recommendations for human epidermal growth factor receptor 2 testing in breast cancer," Archives of Pathology and Laboratory Medicine, vol. 131, no. 1, pp. 18-43, 2007.

[16] M. Vergara-Lluri, N. Moatamed, E. Hong, and S. Apple, "High concordance between HercepTest immunohistochemistry and ERBB2 fluorescence In situ hybridization before and after implementation of American Society of Clinical Oncology/College of American Pathology 2007 guidelines," Modern Pathology, vol. 25, pp. 1326-1332, 2012.

[17] J. S. Ross, "Fluorescence In situ hybridization is the preferred approach over immunohistochemistry for determining HER2 status," Clinical Chemistry, vol. 57, no. 7, pp. 980-982, 2011.

[18] G. Sauter, J. Lee, J. Bartlett, D. Slamon, and M. Press, “Guidelines for human epidermal growth factor receptor 2 testing: biologic and methodologic considerations," Journal of Clinical Oncology, vol. 27, no. 8, pp. 1323-1333, 2009.

[19] E. E. Grimm, R. A. Schmidt, P. E. Swanson, S. M. Dintzis, and K. H. Allison, "Achieving 95\% cross-methodological concordance in HER2 testing: causes and implications of discordant cases," American Journal of Clinical Pathology, vol. 134, no. 2, pp. 284292, 2010.

[20] W. Hanna, P. Barnes, R. Berendt et al., "Testing for Her2 in breast cancer: current pathology challenges faced in Canada," Current Oncology, vol. 19, pp. 315-323, 2012.

[21] C. Franchet, T. Filleron, A. Cayre et al., "Instant-quality fluorescence in-situ hybridization as a new tool for HER2 testing in breast cancer: a comparative study," Histopathology, vol. 64, no. 2, pp. 274-283, 2013.

[22] A. Mohamed, K. Krajewski, B. Cakar, and C. X. Ma, "Targeted therapy for breast cancer," The American Journal of Pathology, vol. 183, no. 4, pp. 1096-1112, 2013. 


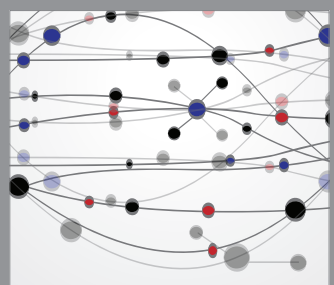

The Scientific World Journal
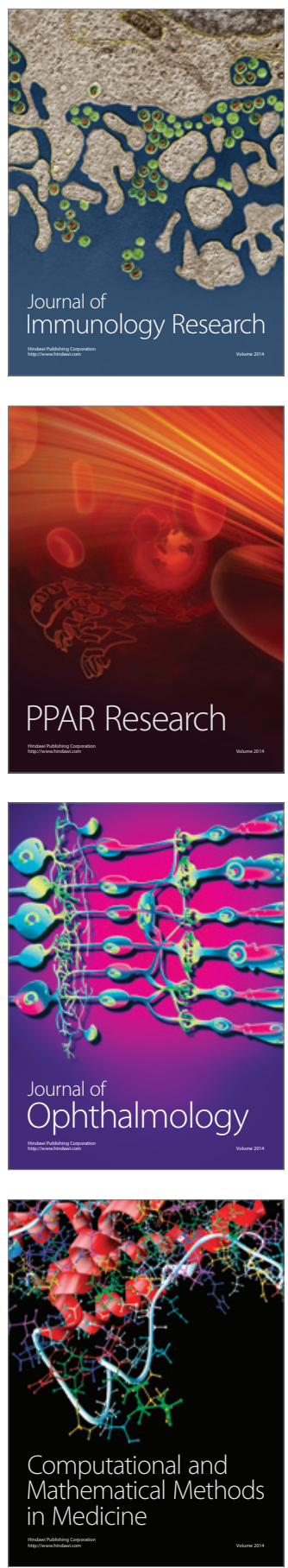

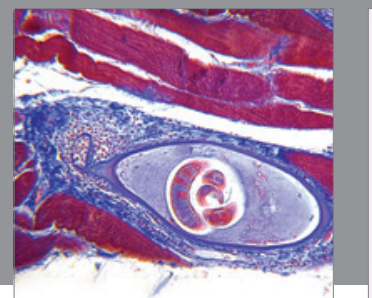

Gastroenterology

Research and Practice
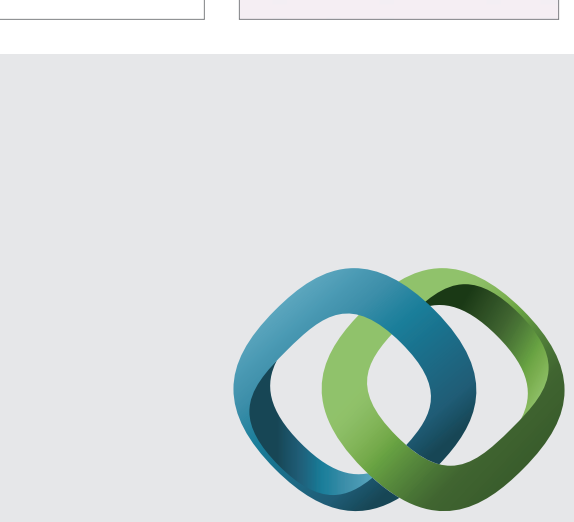

\section{Hindawi}

Submit your manuscripts at

http://www.hindawi.com
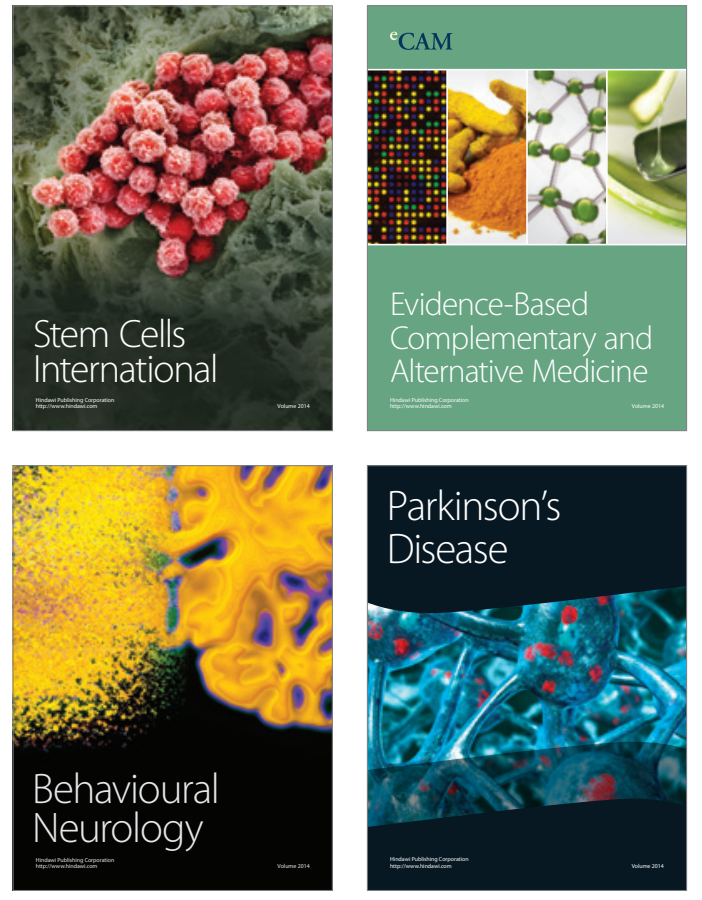
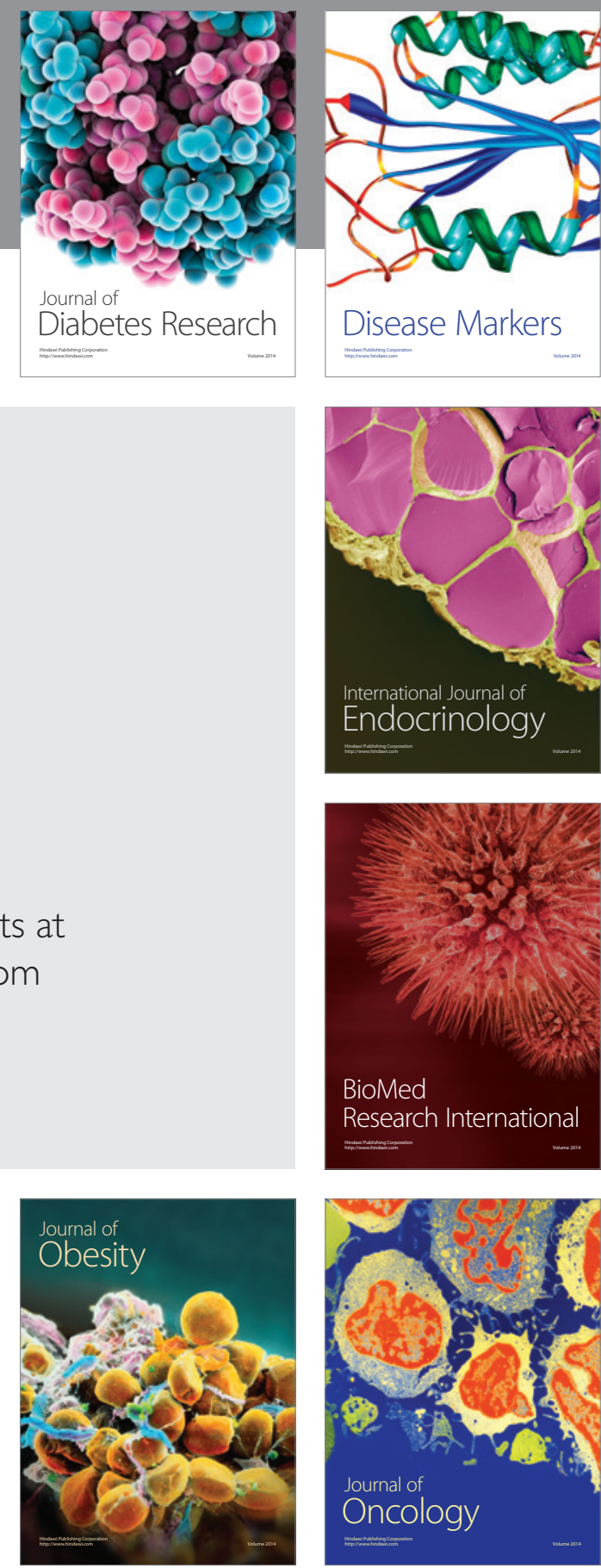

Disease Markers
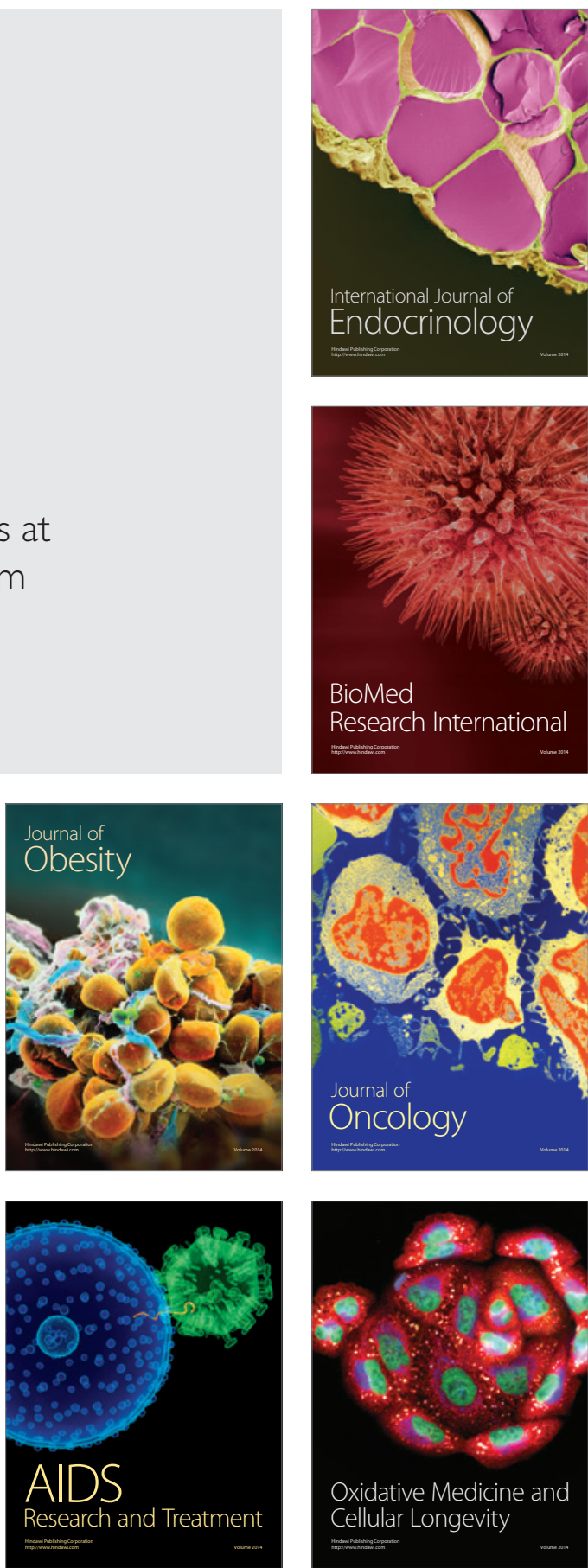